\title{
Tacrolimus downregulates inflammation by regulating pro-/anti-inflammatory responses in LPS-induced keratitis
}

\author{
YIFENG YU ${ }^{1 *}$, JING ZHONG $^{2 *}$, LULU PENG $^{2}$, BOWEN WANG ${ }^{2}$, SAIQUN LI $^{2}$, HAIXIANG HUANG $^{2}$, \\ YUQING DENG ${ }^{2}$, HENAN ZHANG ${ }^{2}$, RUHUI YANG ${ }^{2}$, CHANGYUN WANG $^{1}$ and JIN YUAN ${ }^{2}$ \\ ${ }^{1}$ Department of Ophthalmology, The Second Affiliated Hospital of Nanchang University, Nanchang, Jiangxi 330006; \\ ${ }^{2}$ State Key Laboratory of Ophthalmology, Zhongshan Ophthalmic Center, \\ Sun Yat-Sen University, Guangzhou, Guangdong 510064, P.R. China
}

Received January 24, 2017; Accepted July 12, 2017

DOI: $10.3892 / \mathrm{mmr} .2017 .7353$

\begin{abstract}
Lipopolysaccharide (LPS)-induced keratitis is a progressive infectious ocular disease in which innate inflammatory responses often cause clinical tissue damage and vision loss. The present study aimed to investigate the effects of tacrolimus, an effective immunomodulator, on LPS-induced innate immune responses. The effects of tacrolimus on the apoptotic rate and viability of human corneal epithelial cells (HCECs), polymorphonuclear neutrophils (PMNs) and monocytes (THP-1 cells) were examined using flow cytometry and MTT assays. Subsequently, the role of tacrolimus on LPS-induced inflammation in HCECs, PMNs and THP-1 cells was evaluated by detecting the expression levels of pro-inflammatory cytokines, including interleukin (IL)-1 $\beta$, IL-6 and matrix metallopeptidase 9; anti-inflammatory cytokines, including IL-10 and transforming growth factor- $\beta$; and proangiogenic factors, including vascular endothelial growth factor and tumor necrosis factor- $\alpha$ using quantitative polymerase chain reaction. The results demonstrated that tacrolimus had good biocompatibility with HCECs, while promoting apoptosis and decreasing the viability of PMNs and THP-1 cells. Furthermore, tacrolimus effectively reduced the expression levels of pro-inflammatory cytokines and increased anti-inflammatory cytokines in LPS-induced keratitis in vitro. Notably, tacrolimus decreased the levels of proangiogenic factors, which are highly increased following LPS stimulation. Conclusively, tacrolimus appears to be a safe and effective treatment to suppress neutrophil and monocyte activity, modulate the balance of pro-/anti-inflammatory cytokines,
\end{abstract}

Correspondence to: Dr Jin Yuan, State Key Laboratory of Ophthalmology, Zhongshan Ophthalmic Center, Sun Yat-Sen University, 54 Xianlie South Road, Guangzhou, Guangdong 510064, P.R. China

E-mail: yuanjincornea@126.com

*Contributed equally

Key words: tacrolimus, pro-/anti-inflammatory responses, LPS, keratitis and reduce the inflammatory response and angiogenic activity in LPS-induced bacterial keratitis.

\section{Introduction}

Microbial keratitis is a common ocular infection caused by bacteria, fungi, viruses or parasites and is the second most significant cause of monocular blindness, particularly in certain developing countries and, generally, in the tropics (1). Clinically, this infection requires aggressive antimicrobial management to eliminate the causative organisms, suppress destructive reactions, and restore normal ocular structure and vision $(2,3)$. However, despite timely and correct therapeutic strategies, infective keratitis remains clinically challenging, in which approximately $50 \%$ of eyes have poor visual outcomes $(4,5)$, because conventional therapies, such as antibiotic treatment, often fail to control the tissue damage caused by excessive local inflammation, even if viable bacteria are cleared from the cornea (6). Hence, in addition to antibiotic treatment, it is also important to develop new therapeutic modalities to control the inflammatory response in microbial keratitis.

The innate immune response is the first line of host defence that invading pathogens rely on for innate immune recognition through pattern recognition receptors (PRRs) (7). The activation of PRRs initiates a variety of inflammatory events, including the infiltration of inflammatory cells (e.g., polymorphnuclear neutrophils [PMNs] and monocytes/macrophages), the production of pro- and anti-inflammatory cytokines (7), the interplay of cellular apoptosis vs. necrosis (8), and causes immune cells to become refractory to subsequent endotoxin challenge a state known as endotoxin tolerance (9). These PRR-mediated inflammatory responses are necessary for microbial clearance; however, if uncontrolled, excessive host inflammation also leads to immunopathological tissue damage (7). Therefore, it is important to precisely balance proand anti-inflammatory responses in ocular immune defence.

Tacrolimus (FK506), a macrolide molecule that suppresses the activation of T cells, T helper cell-mediated B-cell proliferation and the formation of cytokines $(10,11)$, the reports of its role on non-T cells is comparatively few but it has been reported that FK506 cannot control the cytokine production 
in non-T cells in cornea transplantation (12). It has been widely used clinically in preventing organ transplant rejection and autoimmune diseases (13-15) and has also been effective in preventing corneal transplant rejection (16), dry eye (18), and steroid-resistant refractory VKC (17) in ophthalmology. Recently, Erdal demonstrated that FK506 causes a significant decrease in inflammatory cells accompanied by reduced corneal vascularisation in the experimental herpetic stromal keratitis corneal edema model (18). Moreover, our previous study demonstrated that tacrolimus can inhibit the inflammation induced by fungi and alleviate the severity of corneal damage at an early stage of fungal keratitis (19); and Wang et al (20) showed that a high dose of FK506 promoted HEV infection. These studies together suggest that tacrolimus is a good immunoregulator in viral and fungal inflammatory responses. Furthermore, it has been reported that FK506 cannot control the cytokine production in non-T cells in cornea transplantation (12) while its role on non-T cells in bacterial keratitis remains unknown; however, its role in bacterial keratitis has few reports, especially its role on non-T cells, and many aspects remain unknown.

Microbial keratitis induced by lipopolysaccharide (LPS), a well-characterized pathogen-associated molecular pattern found in the outer leaflet of the outer membrane of the bacteria, is a rapidly progressive infectious ocular disease (21). Upon recognition of invading microbes by PRRs, inflammatory cells are recruited to the cornea to produce various pro-inflammatory cytokines (22) (e.g., IL-6 and IL-1 $\beta$ ) and modulate anti-inflammatory cytokines (23) (e.g., IL-10 and TGF- $\beta$ ) to regulate antibacterial immunity (24). However, if uncontrolled, these inflammatory mediators often elicit an overly robust response, resulting in bystander tissue damage. Thus, tight regulation of innate immune response, especially pro-inflammatory and anti-inflammatory responses, is critical for the resolution of LPS-induced bacterial keratitis (21).

With this background, the present study was aimed to explore the role of tacrolimus in LPS-induced bacterial keratitis. An in vitro study was designed to explore the histocompatibility and immunoregulation of tacrolimus in human corneal epithelial cells, PMN, and monocytes after LPS stimulation.

\section{Materials and methods}

Cell culture and isolation of human PMN. Human monocytic THP-1 cells (ATCC-TIB-202, Manassas, VA, USA) were cultured in RPMI-1640 medium supplemented with $10 \%$ fetal bovine serum (FBS) and 1\% penicillin-streptomycin (Invitrogen, Carlsbad, CA, USA). A human corneal epithelial cell (HCEC) line was obtained from American Type Culture Collection (ATCC-CRL-11515; ATCC, Manassas, VA, USA). The cells were maintained in keratinocyte serum-free medium (GIBCO-BRL 17005-042; Gibco, Grand Island, NY, USA) supplemented with $0.05 \mathrm{mg} / \mathrm{ml}$ bovine pituitary extract, $5 \mathrm{ng} / \mathrm{ml}$ human recombinant epidermal growth factor, $0.005 \mathrm{mg} / \mathrm{ml}$ insulin, $500 \mathrm{ng} / \mathrm{ml}$ hydrocortisone, $100 \mathrm{IU} / \mathrm{ml}$ penicillin, $100 \mathrm{lg} / \mathrm{ml}$ streptomycin, and $0.125 \mathrm{lg} / \mathrm{ml}$ amphotericin B.

The fresh isolation of human PMN, using blood of 3 healthy male and 3 healthy female volunteers (ages 30-45), was performed as previously described with minor
Table I. Nucleotide sequences of the specific primers used for PCR amplification.

\begin{tabular}{ll}
\hline Gene & \multicolumn{1}{c}{ Primer Sequence (5'-3') } \\
\hline IL-1 $\beta$ & F: TGTATGTGACTGCCCAAGAT \\
& R: GCACACCCAGTAGTCTTGCT \\
IL6 & F: GCACTGGCAGAAAACAACCT \\
& R: GCTCTGGCTTGTTCCTCACTAC \\
MMP9 & F: GAAAGCCTATTTCTGCCAGG \\
& R: TGCAGGATGTCATAGGTCAC \\
IL-10 & F: AGCTGGACAACATACTGCTAACCGAC \\
& R: CTTGATTTCTGGGCCATGCTTCTCTG \\
TGF- $\beta$ & F: GCAGCTGTACATTGACTTC \\
& R: GTTATGCTGGTTGTACAGGG \\
VEGF & F: GCAGATTATGCGGATCAAACC \\
& R: TTTCGTTTTGCCCCTTTCC \\
TNF- $\alpha$ & F: GGTATGAGCCCATCTATC TG \\
& R: GCAATGATCCCAAAGTAGAC \\
3 -actin & F: GCTCCTCCTGAGCGCAAG \\
& R: CATCTGCTGGAAGGTGGACA
\end{tabular}

modifications (25). In short, blood was diluted with cold HBSS (1:1), after which, gradient centrifugation was used to remove the lymphocytes. The remaining lowest layer containing PMN and erythrocytes was then suspended in cold lysis buffer to lyse the erythrocytes. The remaining pellet containing only PMN was suspended in HBSS, and the cells were counted using a Bürker chamber. Viability was evaluated using trypan blue dye exclusion (0.4\%). Keeping all solutions and PMN on ice to prevent premature activation, this isolation method consistently yielded PMN with a viability $>95 \%$. The cells were cultured at $37^{\circ} \mathrm{C}$ in a humidified atmosphere with $5 \%$ carbon dioxide. The culture medium was changed daily.

FK506 was firstly dissolved in Dimethylsulfoxide (DMSO) with a concentration of $10 \mathrm{mg} / \mathrm{ml}(1 \%)$, and then was incubated with culture medium containing FK506 at a final concentration of $0.1 \%$.

Flow cytometry. Tacrolimus' role on the apoptosis of THP-1, HCEC and PMN was determined by flow cytometry, and each cell was divided into two groups: Group I was the control group and received no treatment; and group II received 0.1\% FK506 (Sigma-Aldrich, St. Louis, MO, USA) for $24 \mathrm{~h}$.

Cells were seeded at $5 \times 10^{5}$ cells/well in 12 -well plates, and the Annexin V-fluorescein isothiocyanate Apoptosis Detection kit (BD Bioscience, CA, USA) was used according to the manufacturer's instructions. Briefly, cells were pooled, washed, and resuspended in $500 \mu \mathrm{l}$ of binding buffer, followed by the addition of $5 \mu \mathrm{l}$ of Annexin V-fluorescein isothiocyanate and $5 \mu \mathrm{l}$ of PI. Then, the cells were incubated at room temperature in the dark for $15 \mathrm{~min}$ and analysed by flow cytometry (EPICS XL/MCL; Beckman Coulter Miami, FL, USA). Viable cells did not exhibit Annexin V or PI staining, early-apoptotic cells showed Annexin V but not PI staining, and late-apoptotic cells exhibited both Annexin V and PI staining. 


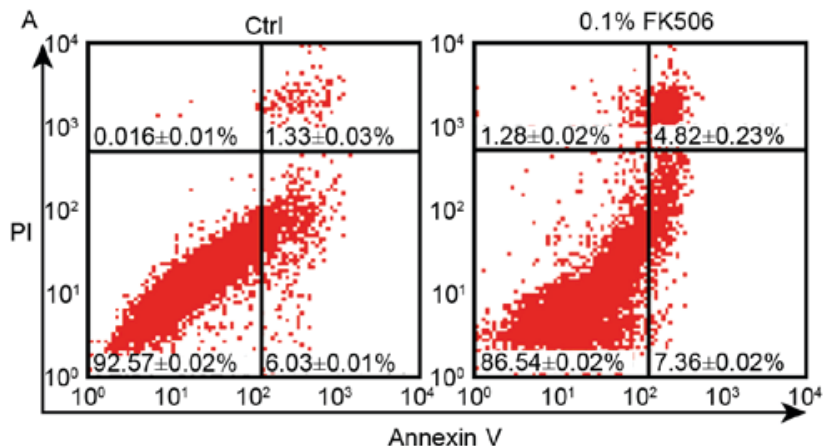

B

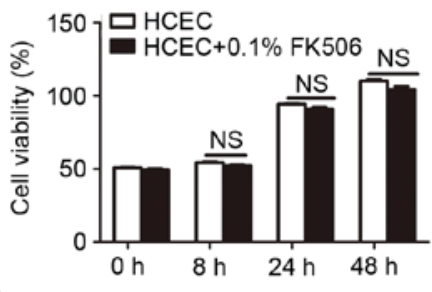

Figure 1. Tacrolimus showed good biocompatibility in HCEC. Flow cytometry showed that FK506 had no negative influences on apoptosis in HCEC (A). The cell viability of HCEC with FK506 was similar to that of the control group at 8, 24 and $48 \mathrm{~h}(\mathrm{~B}) ;{ }^{*} \mathrm{P}<0.05 ;{ }^{* *} \mathrm{P}<0.01 ;{ }^{* * *} \mathrm{P}<0.001$.

Cytotoxicity assay. The cytotoxicity of tacrolimus was evaluated using the MTT assay in THP-1, HCEC and PMN, which were each grouped into four groups: Group I was the control group and received no treatment; group II received $0.1 \%$ tacrolimus for $8 \mathrm{~h}$; group III received $0.1 \%$ tacrolimus for $24 \mathrm{~h}$; and group IV received $0.1 \%$ tacrolimus for $48 \mathrm{~h}$. THP-1, HCEC and PMN were plated at concentrations of $5 \times 10^{3}$ cells/well in 96-well plates. After $24 \mathrm{~h}$, the medium was replaced with $200 \mu \mathrm{l}$ of serum-free medium. The remaining cells were either untreated or incubated with $30 \mu 1$ of $0.1 \%$ FK506 for 8, 24 or $48 \mathrm{~h}$. Next, the medium in each well was replaced with $200 \mu 1$ of fresh serum-free medium. To each well, $20 \mu \mathrm{l}$ of $5 \mathrm{mg} / \mathrm{ml}$ MTT solution in PBS (pH 7.4) was added. Following incubation for $3 \mathrm{~h}$ at $37^{\circ} \mathrm{C}$, the medium was aspirated, and the formazan crystals that had formed in the well were dissolved in $200 \mu \mathrm{l}$ of DMSO. The absorbance of the dissolved formazan crystals in DMSO was recorded at $570 \mathrm{~nm}$ and used to estimate cell viability relative to control cells.

Real-time PCR. We verified tacrolimus' role on LPS-induced inflammatory responses in THP-1, HCEC and PMN, in which each cell type was divided into three groups with a seeding density of $5 \times 10^{6}$ cells/well in 6-well plates: Group I was the control group that received PBS; group II received $5 \mu \mathrm{g} / \mathrm{ml}$ LPS (Sigma-Aldrich) for $8 \mathrm{~h}$; and group III was incubated with $0.1 \%$ FK506 for $24 \mathrm{~h}$ before being treated with $5 \mu \mathrm{g} / \mathrm{ml}$ LPS for $8 \mathrm{~h}$. Total RNA was isolated from cultured cells using TRIzol (Invitrogen) according to the manufacturer's recommendations and was quantified using a NanoDrop 2000C spectrophotometer (Thermo Scientific, West Palm Beach, FL, USA). One microgram of total RNA was reverse transcribed to produce cDNA, and the cDNA was amplified using SYBR-Green Master Mix (Bio-Rad Laboratories, Inc., Hercules, CA, USA) according to the manufacturer's instructions. Primers for human pro-inflammatory cytokines, such as IL-1 $\beta$, IL-6 and MMP9, human anti-inflammatory cytokines, such as IL-10 and TGF- $\beta$, and human angiogenesis factors, such as VEGF and TNF- $\alpha$ were purchased from SABiosciences (Frederick, MD, USA), and the primer sequences are listed in Table I. Quantitative real-time PCR was performed using a CFX96 Real-Time PCR System (Bio-Rad Laboratories, Inc.). Relative gene expression levels were calculated after normalization to the internal control $\beta$-actin.
Statistical analyses. Unpaired, two-tailed Student's t test was used to determine the significance of the flow cytometry and MTT assays. Differences in the RNA expression levels of pro-inflammatory, anti-inflammatory and angiogenesis cytokines in the three groups were determined using the Mann-Whitney U test. The data were considered significant at $\mathrm{P}<0.05$.

\section{Results}

The cytotoxicity of tacrolimus on HCEC. To confirm the safety of tacrolimus used in the in vitro experiment, HCEC were used to determine the biocompatibility using flow cytometry and MTT assays. The results demonstrated that tacrolimus had no obvious influence on apoptosis; early and late apoptosis in the control and tacrolimus groups showed no significant differences ( $\mathrm{P}>0.05)$ (Fig. 1A). After incubation for 8, 24 and $48 \mathrm{~h}$, HCEC viability showed a slight reduction, with no significant difference $(\mathrm{P}>0.05)$ (Fig. 1B). The results demonstrated that tacrolimus (FK506) had good biocompatibility in HCEC.

Tacrolimus increased apoptosis and decreased the viability of THP-1 and PMN cells. To explore the role of tacrolimus on the proliferation of two main human inflammatory cells, $0.1 \%$ FK506 was added to THP-1 and PMN to analyse the apoptosis rate and cell viability compared with that of the control group. In PMN, flow cytometry (Fig. 2A) showed that the early and the late-apoptosis rate was $50.2 \pm 1.02$ and $35.9 \pm 3.71 \%$, respectively, in the FK506 group after $24 \mathrm{~h}$, which was significantly higher than that of the control group with $5.36 \pm 0.11 \%(\mathrm{P}<0.05)$ and $18.66 \pm 0.27 \%(\mathrm{P}<0.05)$. Moreover, we incubated cells without or with FK506 for 8, 24 and 48 h, and evaluation of cell viability revealed that FK506 had a significant inhibitory effect on cell viability, which correlated positively with the stimulation time (Fig. 2B). In THP-1, for both early or late apoptosis, the rates in the FK506 group after $24 \mathrm{~h}, 88.43 \pm 0.23$ and $2.71 \pm 0.12 \%$, respectively, were greater than the rates in the control group, which had a high fraction of normal cells $96.04 \pm 0.03 \%$ and a low fraction of early $(1.94 \pm 0.02 \%)(\mathrm{P}<0.01)$ and late $(1.88 \pm 0.01 \%)$ apoptotic cells $(\mathrm{P}<0.01)$ (Fig. 2C). The control group displayed significantly higher cell viability at 8,24 and 48 h than the FK506 group $(\mathrm{P}<0.001)$ (Fig. 2D). Conclusively, FK506 raise the apoptosis's rates and reduced cell viability in THP-1 and PMN. 

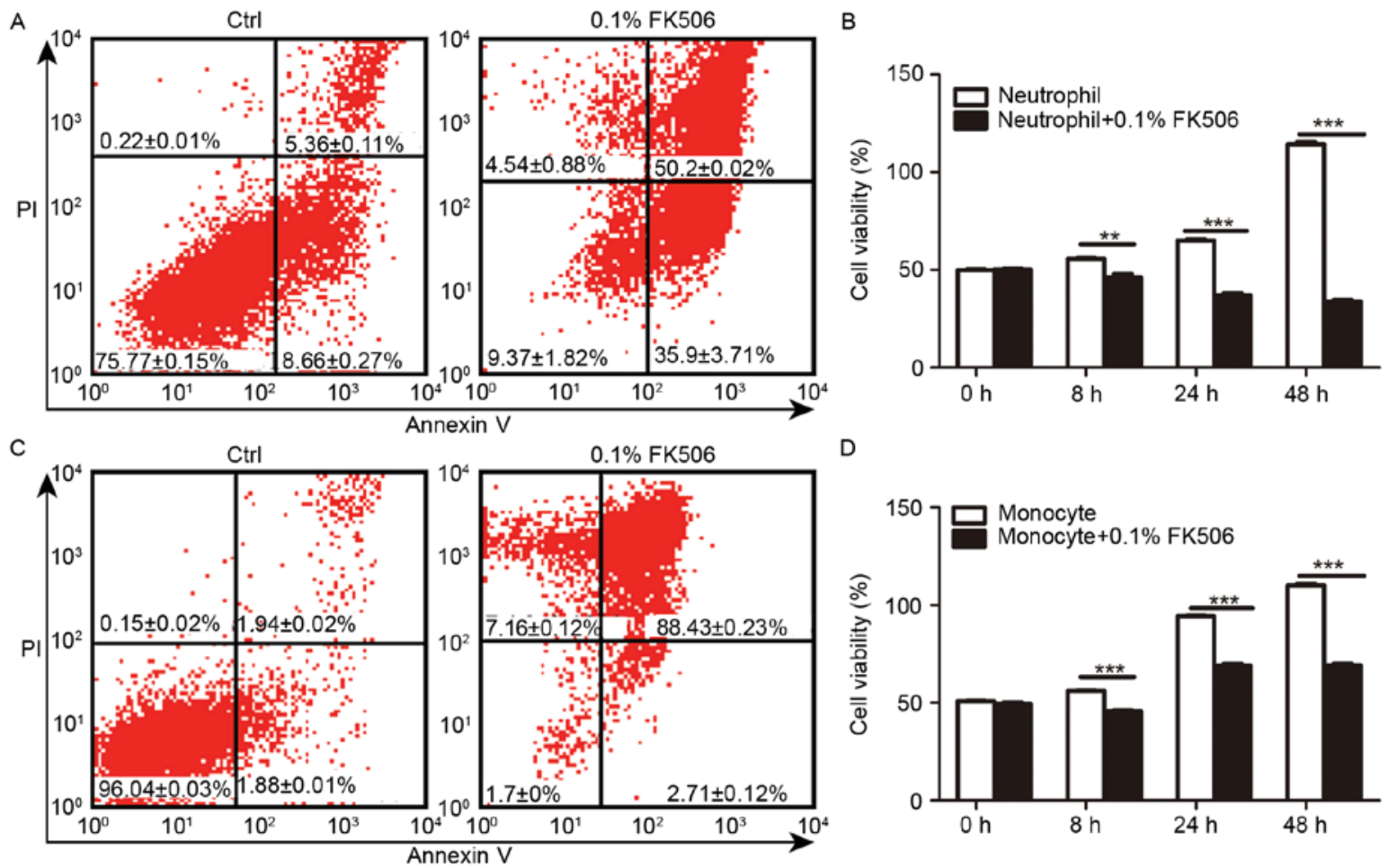

D

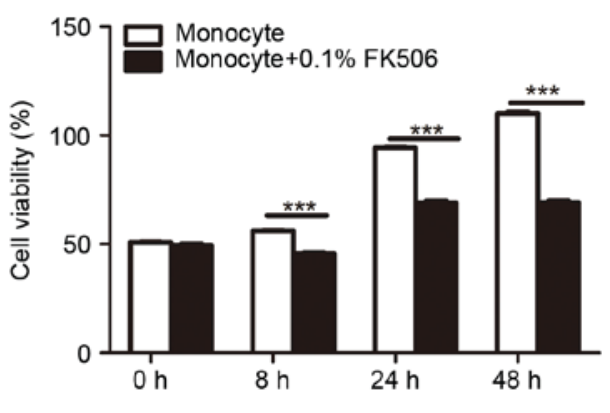

Figure 2. Tacrolimus increased cell apoptosis and decreased cell viability in neutrophils and monocytes by flow cytometry and MTT; $0.1 \%$ FK506 elevated the fraction of neutrophils in early and late apoptosis after $24 \mathrm{~h}$ (A) and reduced cell viability at 8,24 and $48 \mathrm{~h}$ (B). The apoptosis of monocytes in the $0.1 \%$ FK506 group was reduced $(\mathrm{C})$, and cell viability was reduced at 8,24 and $48 \mathrm{~h}(\mathrm{D}) ;{ }^{*} \mathrm{P}<0.05 ;{ }^{* * *} \mathrm{P}<0.01 ;{ }^{* * * *} \mathrm{P}<0.001$.
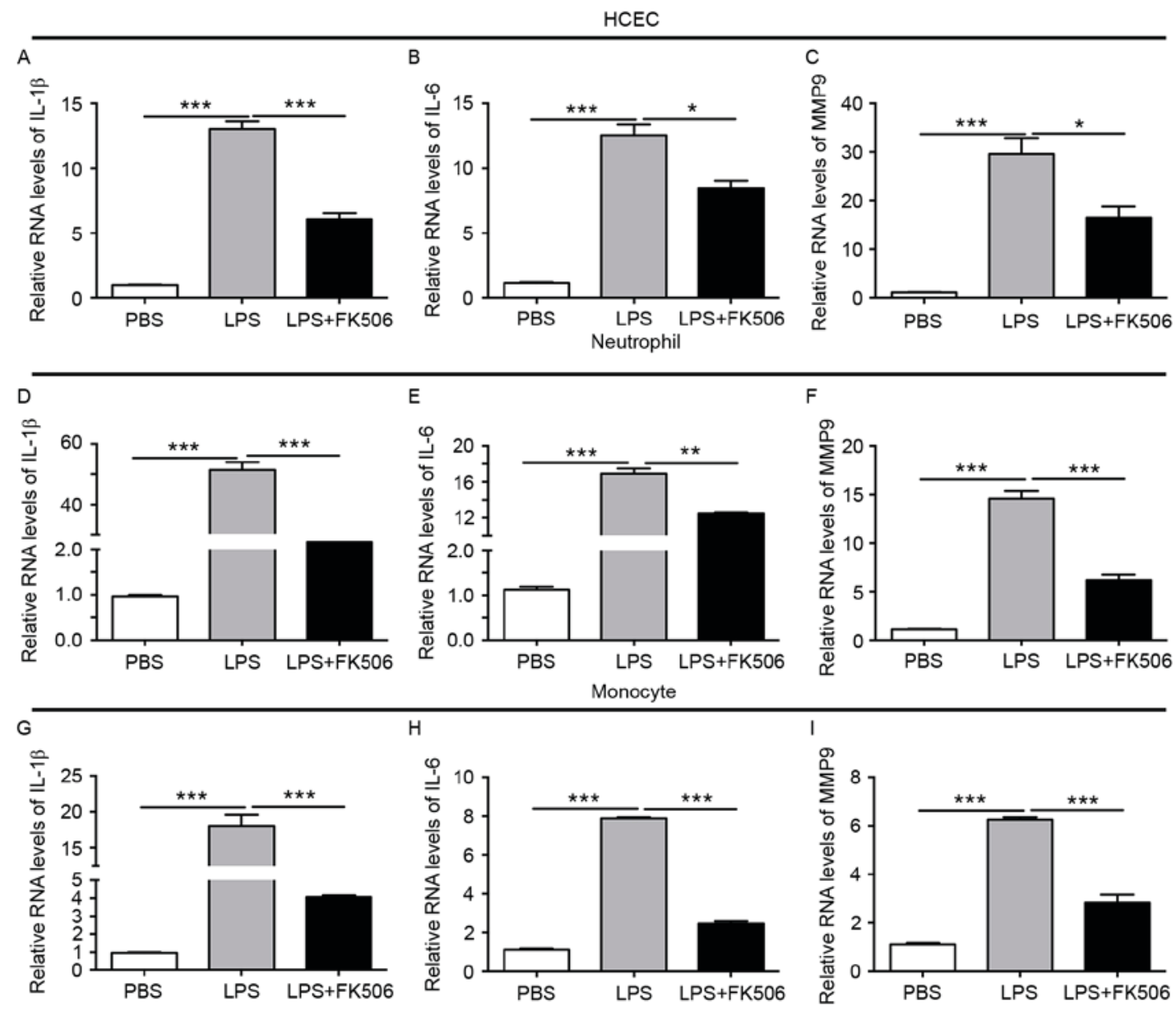

Figure 3. Tacrolimus reduced the pro-inflammatory cytokines in LPS-induced inflammatory responses by real-time PCR; $0.1 \%$ FK506 inhibited the expression levels of IL-1 $\beta$, IL-6 and MMP9 in HCEC (A-C), neutrophils (D-F) and monocytes (G-I) after LPS stimulation for $8 \mathrm{~h} ;{ }^{*} \mathrm{P}<0.05 ;{ }^{* *} \mathrm{P}<0.01 ;{ }^{* * *} \mathrm{P}<0.001$ 

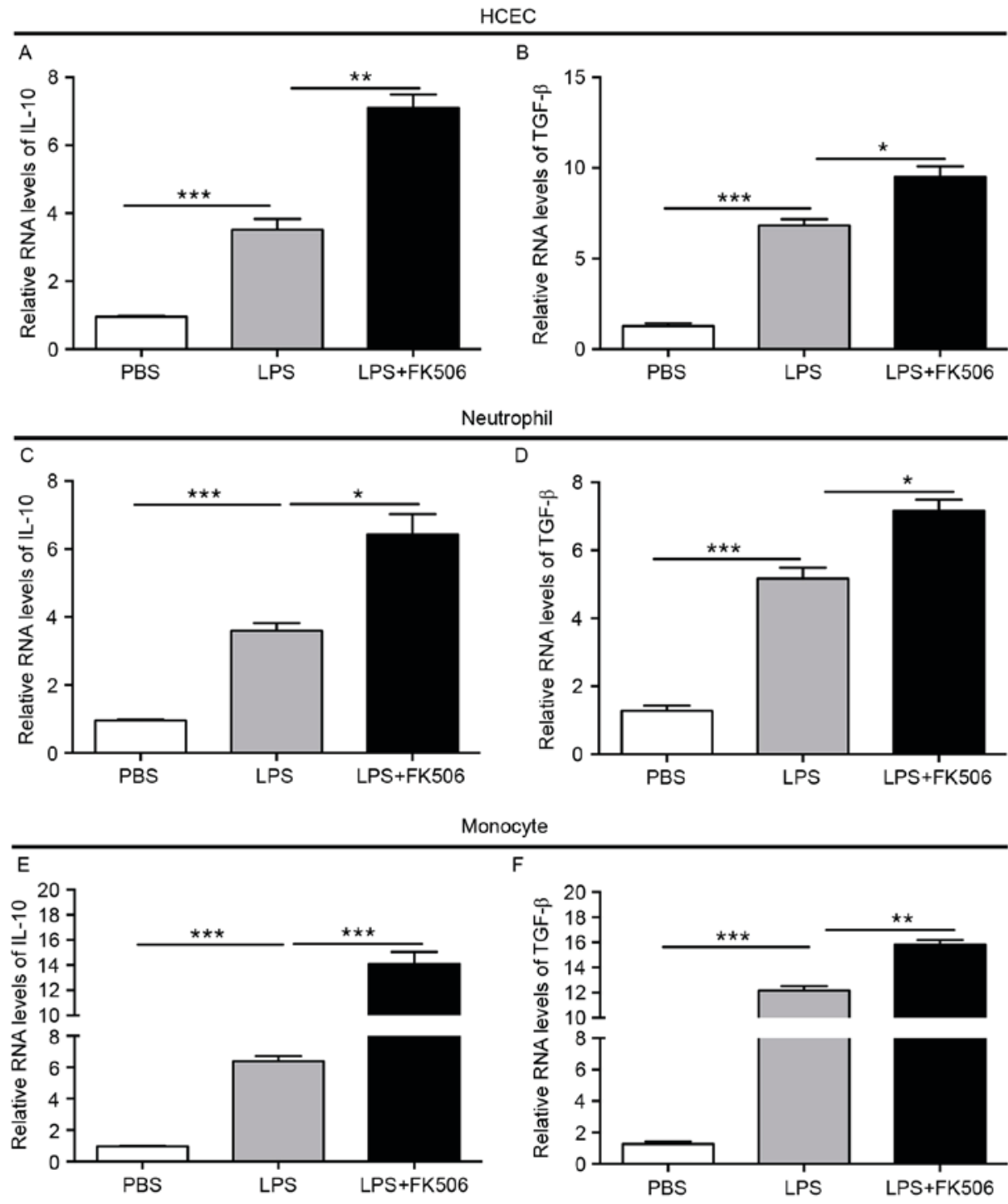

Figure 4. Tacrolimus increased the anti-inflammatory cytokines in LPS-induced inflammatory responses by real-time PCR; $0.1 \%$ FK506 promoted the expression levels of IL-10 and TGF- $\beta$ in HCEC (A and B), neutrophils (C and D) and monocytes (E and F) after LPS stimulation for 8 h; ${ }^{*} \mathrm{P}<0.05 ;{ }^{* * *} \mathrm{P}<0.01{ }^{* * * *} \mathrm{P}<0.001$.

Tacrolimus reduced pro-inflammatory cytokines in LPS-induced inflammatory responses. Based on the decreased apoptosis and cell viability in HCEC, THP-1 and PMN, we further evaluated tacrolimus' role on the pro-inflammatory cytokines in LPS-induced inflammatory responses. In HCEC with LPS, IL-1 $\beta$, and IL- 6 increased up to approximately 10 times, and MMP-9 to 20 times more than the control group (both $\mathrm{P}<0.001$ ), while after the FK506 intervention, both expression levels obviously decreased (both $\mathrm{P}<0.05$ ) (Fig. 3A-C). In PMN, after incubation with LPS for $8 \mathrm{~h}, \mathrm{IL}-1 \beta$, IL-6 and MMP9 showed a clear elevation (both $\mathrm{P}<0.001$ ), while in the LPS + FK506 group, IL-1 $\beta$ reduced its expression to approximately one thirtieth of the LPS group $(\mathrm{P}<0.001)$, IL-6 was reduced by one third $(\mathrm{P}<0.01)$, and MMP9 was also lower than the LPS group $(\mathrm{P}<0.001)$ (Fig. 3D-F). In monocytes, compared with the control group, pro-inflammatory cytokines also displayed a similar tendency to increase after stimulation with LPS; moreover, FK506 depressed the expression of IL-1 $\beta$, IL-6 and MMP9 (both P<0.001) (Fig. 3G-I). Therefore, we concluded that FK506 alleviated the expression of pro-inflammatory cytokines IL-1 $\beta$, IL-6 and MMP9 induced by LPS.
Tacrolimus increased anti-inflammatory cytokines in LPS-induced inflammatory responses. Furthermore, we detected the levels of the anti-inflammatory cytokines IL-10 and TGF- $\beta$ in LPS-induced inflammatory responses. In HCEC, IL-10 and TGF- $\beta$ showed mild elevation after LPS stimulation, and a more obvious significant upregulation could be observed in the LPS + FK506 group (both $\mathrm{P}<0.05$ ) (Fig. 4A-B). Compared with the control group, IL-10 and TGF- $\beta$ levels gradually increased 4 to 5 times in the neutrophil LPS group (both $\mathrm{P}<0.001$ ), then showed a continuous increase after treatment with FK506 (both P<0.05) (Fig. 4C-D). For monocytes, LPS also improved the expression of IL-10 and TGF- $\beta$, and FK506 significantly enhanced their levels, where IL-10 was almost 2 times more in the LPS + FK506 group than the LPS group $(\mathrm{P}<0.001)$, and TGF- $\beta$ was slightly higher than the LPS group $(\mathrm{P}<0.01)$ (Fig. 4E-F). These results suggest that FK506 partially improved anti-inflammatory cytokine levels after the inflammatory cytokines were induced by LPS.

Tacrolimus lessened proangiogenic factors in LPS-induced inflammatory responses. VEGF and TNF- $\alpha$ are important 

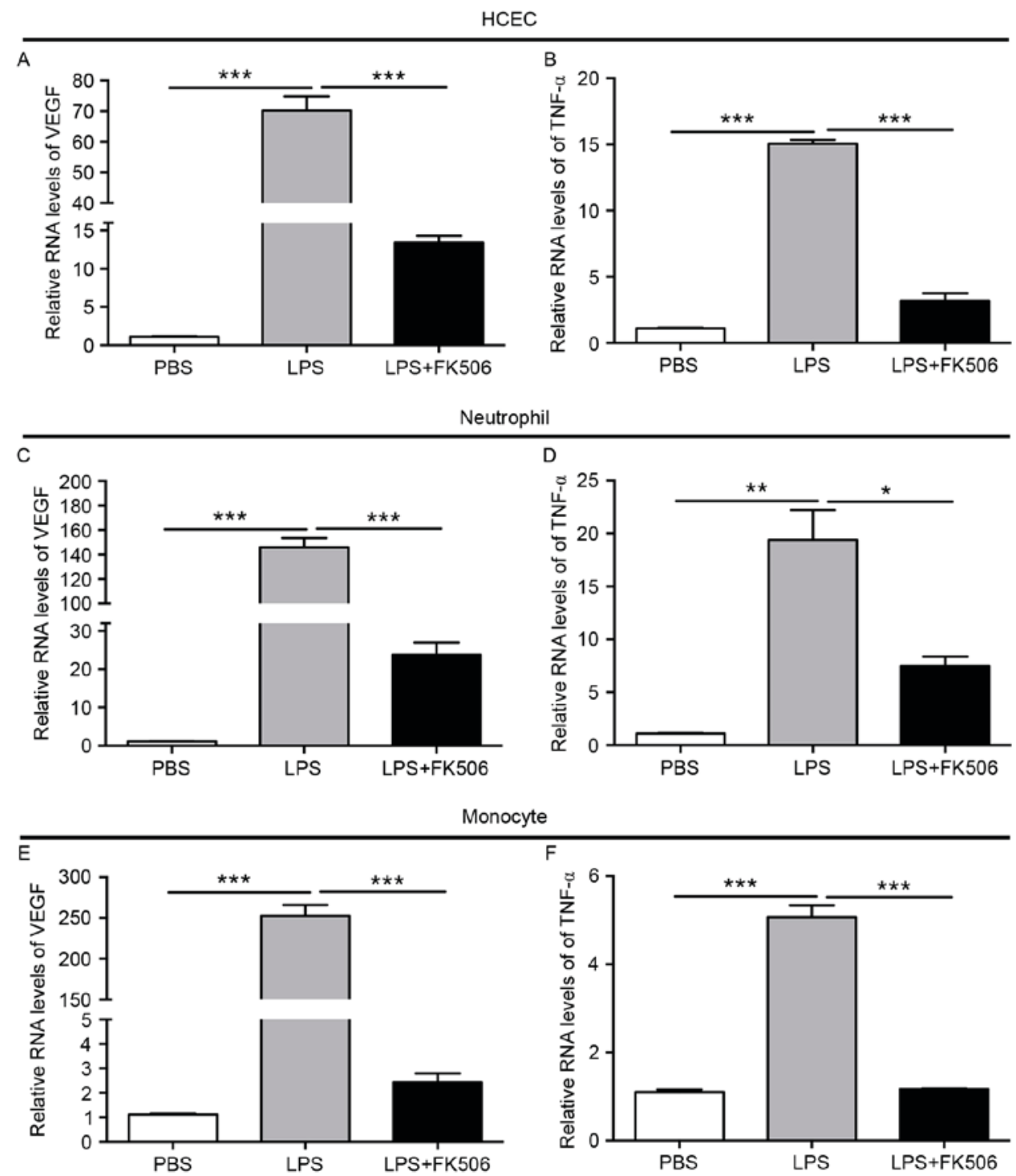

Figure 5. Tacrolimus had a negative role in the proangiogenic factors in LPS-induced inflammatory responses by real-time PCR; $0.1 \%$ FK506 downregulated the expression of VEGF and TNF- $\alpha$ in HCEC (A and B), neutrophils (C and D) and monocytes (E and F) after LPS stimulation for $8 \mathrm{~h} ;{ }^{*} \mathrm{P}<0.05 ;{ }^{* *} \mathrm{P}<0.01 ;{ }^{* * * *} \mathrm{P}<0.001$.

angiogenesis factors. In HCEC with LPS, compared with the control group, VEGF increased approximately 70 times $(\mathrm{P}<0.001)$, which was then reduced to approximately 10 times after FK506 $(\mathrm{P}<0.001)$. TNF- $\alpha$ was slightly elevated in the LPS group $(\mathrm{P}<0.001)$, and then declined drastically in the LPS + FK506 group $(\mathrm{P}<0.001)$ (Fig. 5A-B). Incredibly, compared with the control group, in PMN, VEGF levels increased rapidly by approximately 150 times in the LPS group $(\mathrm{P}<0.001)$, then significantly decreased to approximately 20 times by FK506 $(\mathrm{P}<0.001)$; in addition, TNF- $\alpha$ levels were also increased in the LPS group and decreased by FK506 (Fig. 5C-D). With regard to monocytes, VEGF also exhibited an apparent upregulation after LPS $(\mathrm{P}<0.001)$ and doubled that of the control group. A slight enhancement was observed in TNF- $\alpha$ in the LPS group $(\mathrm{P}<0.001)$, which lessened to almost the same levels as the control group in the LPS + FK group (Fig. 5E-F). Taken together, these results clearly demonstrated that the angiogenesis factors VEGF and TNF- $\alpha$ were markedly increased after LPS induction in HCEC, PMN and THP1 and that FK506 was partly effective in reducing these levels.

\section{Discussion}

In microbial keratitis, there is activation of the immune system, in which inflammatory cells are involved in the cornea's inflammatory response to microbial proliferation and invasion (26); these host reactions account for much of the oedematous, infiltrative, and necrotizing changes (27). Both pro-inflammatory and anti-inflammatory processes involve chemoattractants, adhesion molecules, and other mediators (27). However, this inflammatory response of the immune system is a double-edged sword in the host defence mechanism. On one hand, this response is the main approach that the immune system uses to eliminate pathogens, which is beneficial in protecting against microbial infection. On the other hand, overenthusiastic inflammation can be damaging, and persistent inflammation can lead to degeneration or tissue necrosis (28). In fungal keratitis, a type of severe microbial keratitis, excessive inflammation is an important contributor to corneal damage during fungal infection. Our previous study demonstrated that tacrolimus exerted an obvious 
anti-inflammatory effect by not only reducing the infiltration of inflammatory cells but also suppressing the expression of pro-inflammatory cytokines in innate immune responses at an early stage of corneal fungal infection (21).

In LPS-induced bacterial keratitis, the innate immune responses are important, not just for protecting the host during the period that adaptive responses are required to develop but also because they can influence the nature of the adaptive response $(24,29)$. The interplay of these two systems also appears to participate in the host response to bacterial corneal infection (30). Clinically, topical antibiotics are mandatory for the treatment of bacterial keratitis; moreover, the most widely used anti-inflammatory agents are corticosteroids, which can regulate the innate and adaptive immune responses by inhibiting leukocytes, downregulating pro-inflammatory cytokines, affecting metalloproteinase production, and modifying wound healing in the inflamed cornea (31). However, topical corticosteroids have consistently been avoided by many clinicians for fear of causing immunosuppression, which would lead to neutrophil inhibition, accelerated bacterial replication and an exacerbated infection in acute inflammation $(32,33)$. While clinically, even if the causative organisms are eradicated by antibiotics, the excessive inflammation would also deteriorate the clinical features; therefore, except for corticosteroids, exploring other adjuvant therapies for bacterial keratitis is important. In the present study, we studied the topical immunosuppressant tacrolimus to evaluate its role in modulating pro-/anti-inflammatory responses in LPS-induced bacterial keratitis.

Although the inhibitory mechanisms of tacrolimus have been extensively studied in T cells, little is known regarding the precise suppressive mechanisms of FK506 in non-T cells. We found that tacrolimus induced the apoptosis of PMN and monocytes, which was mainly early apoptosis. Among these two cell types, the early- and late-apoptosis rate of monocytes was consistent with Yoshino's report, which also determined that tacrolimus suppresses the function of macrophages and promotes their apoptosis (34). Moreover, the results showed that both pro- and anti-inflammatory cytokines were activated, and demonstrated that the dynamic balance of pro- and anti-inflammatory responses proposed by Morris et al (35) was disrupted by LPS stimulation in innate immune responses. Then, tacrolimus exhibited its strong regulatory role on reducing pro-inflammatory cytokines and increasing anti-inflammatory cytokines. However, there is evidence that FK506 inhibits T-cell proliferation and T-cell-derived cytokines (36), then modulates lymphocytes, producing less TNF- $\alpha$ and other cytokines, and finally inflammatory responses $(18,37)$. Based on the results of our study, we speculate that tacrolimus may act not only on $\mathrm{T}$ cells but also on PMN and monocytes to modulate their proliferation and their production of inflammatory cytokines and inflammatory responses in LPS-induced inflammation or bacterial keratitis. It is interesting to find that only part and not all of the expression levels of pro- and anti-inflammatory cytokines were modulated by tacrolimus in LPS-induced keratitis, which hints at other pathways other than only innate immune responses participating in this inflammatory activity. Therefore, future research should focus on simultaneously modulating the innate and adaptive immune systems to determine the inflammatory responses in LPS-induced keratitis.
Another important finding in our study is that tacrolimus can reduce LPS-induced angiogenesis in vitro. Published reports have demonstrated that LPS leads to the upregulation of VEGF and promotes angiogenesis (38) and that systemic and topical administration of tacrolimus may be beneficial in the prevention of corneal neovascularization because of its effect on VEGF $(39,40)$. Our study first verified that VEGF and TNF- $\alpha$ were significantly elevated after LPS stimulation in HCEC, PNM and THP-1 and that tacrolimus effectively reduced the expression of VEGF and TNF- $\alpha$ in LPS-induced inflammation or bacterial keratitis. As LPS stimulate macrophages mediators and increase the levels of TNF $\alpha$ through attenuate both FAK and Src activation in osteoblast, which Calcineurin FAK and Src may have been functionally formed a complex $(41,42)$. A possible mechanism in the prevention of VEGF by FK506 in bacterial keratitis might be via the suppression of inflammatory cells and/or a decrease in the levels of the cytokines regulating inflammation (39).

Furthermore, as tacrolimus has not only been regarded as systematic administration (43), but also has been used to suppress immune reactions in chronic disorders, including corneal and limbal transplants, allergic eye disease and uveitis as a long-term use medicine (17), it is thus necessary to confirm the safety of tacrolimus. In our study, 0.1\% FK506 has been demonstrated to be safe in vitro, and no negative influence on apoptosis and cell viability in HCEC was observed. Cell viability was evaluated along with apoptosis, and the results revealed that $0.1 \%$ FK506 had good biocompatibility, with no toxic effects on HCEC. Therefore, ophthalmic tacrolimus is a welcome addition to the therapeutic armamentarium for these corneal and ocular surface diseases, particularly in light of its excellent efficiency and safety profile.

Conclusively, tacrolimus appears to be a safe and effective treatment to suppress the activity of PMN and monocytes, modulate the balance of pro-/anti-inflammatory cytokines, and reduce the inflammatory response and angiogenic activity in LPS-induced bacterial keratitis. Further studies should focus on the specific mechanism of tacrolimus's role in modulating LPS-induced keratitis, and animal models should be used to explore the effects of antibacterial drugs combined with tacrolimus. Further, our study provided references and evidence that FK506 might be a promising adjuvant alternative, together with antibiotics, in treating LPS-induced or bacterial keratitis.

\section{Acknowledgements}

The present study was supported by the National Natural Science Foundation of China to J.Y. (81270972), Guangdong Province Science and Technology Projects to J.Y. (303090100502039) and Jiangxi Province Natural Science Foundation to YF.Y. (20161BAB205270).

\section{References}

1. Chan TC, Agarwal T, Vajpayee RB and Jhanji V: Cross-linking for microbial keratitis. Curr Opin Ophthalmol 27: 348-352, 2016.

2. Keay L, Edwards K, Naduvilath T, Taylor HR, Snibson GR, Forde K and Stapleton F: Microbial keratitis predisposing factors and morbidity. Ophthalmology 113: 109-116, 2006.

3. Wong T, Ormonde S, Gamble G and McGhee CN: Severe infective keratitis leading to hospital admission in New Zealand. Br J Ophthalmol 87: 1103-1108, 2003. 
4. Schaefer F, Bruttin O, Zografos L and Guex-Crosier Y: Bacterial keratitis: A prospective clinical and microbiological study. Br J Ophthalmol 85: 842-847, 2001.

5. Levey SB, Katz HR, Abrams DA, Hirschbein MJ and Marsh MJ: The role of cultures in the management of ulcerative keratitis. Cornea 16: 383-386, 1997.

6. Engel LS, Callegan MC, Hobden JA, Reidy JJ, Hill JM and O'Callaghan RJ: Effectiveness of specific antibiotic/steroid combinations for therapy of experimental Pseudomonas aeruginosa keratitis. Curr Eye Res 14: 229-234, 1995.

7. Hazlett LD: Corneal response to Pseudomonas aeruginosa infection. Prog Retin Eye Res 23: 1-30, 2004.

8. Zhou Z, Barrett RP, McClellan SA, Zhang Y, Szliter EA, van Rooijen N and Hazlett LD: Substance P delays apoptosis, enhancing keratitis after Pseudomonas aeruginosa infection. Invest Ophthalmol Vis Sci 49: 4458-4467, 2008.

9. Biswas SK and Lopez-Collazo E: Endotoxin tolerance: New mechanisms, molecules and clinical significance. Trends Immunol 30: 475-487, 2009.

10. Alaiti S, Kang S, Fiedler VC, Ellis CN, Spurlin DV, Fader D, Ulyanov G, Gadgil SD, Tanase A, Lawrence I, et al: Tacrolimus (FK506) ointment for atopic dermatitis: A phase I study in adults and children. J Am Acad Dermatol 38: 69-76, 1998

11. Kino T, Hatanaka H, Hashimoto M, Nishiyama M, Goto T, Okuhara M, Kohsaka M, Aoki H and Imanaka H: FK-506, a novel immunosuppressant isolated from a Streptomyces. I. Fermentation, isolation, and physico-chemical and biological characteristics. J Antibiot (Tokyo) 40: 1249-1255, 1987.

12. Shao K, Lu Y, Wang J, Chen X, Zhang Z, Wang X, Wang X, Yang $\mathrm{H}$ and Liu G: Different effects of tacrolimus on innate and adaptive immune cells in the allograft transplantation. Scand J Immunol 83: 119-127, 2016.

13. Hannah J, Casian A and D'Cruz D: Tacrolimus use in lupus nephritis: A systematic review and meta-analysis. Autoimmun Rev 15: 93-101, 2016.

14. Ge Y,Zhou H, Shi J, Ye B, Peng Q, Lu X and Wang G: The efficacy of tacrolimus in patients with refractory dermatomyositis/polymyositis: A systematic review. Clin Rheumatol 34: 2097-2103, 2015.

15. Moscovici BK, Holzchuh R, Sakassegawa-Naves FE, Hoshino-Ruiz DR, Albers MB, Santo RM and Hida RY: Treatment of Sjogren's syndrome dry eye using $0.03 \%$ tacrolimus eye drop: Prospective double-blind randomized study. Cont Lens Anterior Eye 38: 373-378, 2015.

16. Fei WL, Chen JQ, Yuan J, Quan DP and Zhou SY: Preliminary study of the effect of FK506 nanospheric-suspension eye drops on rejection of penetrating keratoplasty. J Ocul Pharmaco Ther 24: 235-244, 2008.

17. Kheirkhah A, Zavareh MK, Farzbod F, Mahbod M and Behrouz MJ: Topical $0.005 \%$ tacrolimus eye drop for refractory vernal keratoconjunctivitis. Eye (Lond) 25: 872-880, 2011.

18. Eris E, Yüksel N, Pirhan D, Karadenizli A, Aslan M, Gacar G, Erman G, Subas C, Uzuner H, Yldz DK and Karaöz E: Evaluation of effect of topical Tacrolimus treatment on herpetic stromal keratitis in a rat model. Eye Contact Lens 42: 163-170, 2016.

19. Huang W, Ling S, Jia X, Lin B, Huang X, Zhong J, Li W, Lin X, Sun Y and Yuan J: Tacrolimus (FK506) suppresses TREM-1 expression at an early but not at a late stage in a murine model of fungal keratitis. PLoS One 9: e114386, 2014.

20. Wang Y, Zhou X, Debing Y, Chen K, Van Der Laan LJ, Neyts J, Janssen HL, Metselaar HJ, Peppelenbosch MP and Pan Q: Calcineurin inhibitors stimulate and mycophenolic acid inhibits replication of hepatitis E virus. Gastroenterology 146: 1775-1783, 2014.

21. Chen K, Wu Y, Zhu M, Deng Q, Nie X, Li M, Wu M and Huang X: Lithium chloride promotes host resistance against Pseudomonas aeruginosa keratitis. Mol Vis 19: 1502-1514, 2013.

22. Rocksen D, Koch B, Sandström T and Bucht A: Lung effects during a generalized Shwartzman reaction and therapeutic intervention with dexamethasone or vitamin E. Shock 22: 482-490, 2004

23. Wei H, Yin L, Feng S, Wang X, Yang K, Zhang A and Zhou H: Dual-parallel inhibition of IL-10 and TGF-b1 controls LPS-induced inflammatory response via NF- $\kappa \mathrm{B}$ signaling in grass carp monocytes/macrophages. Fish Shellfish Immunol 44: $445-452,2015$

24. Kerber-Momot T, Leemhuis D, Lührmann A, Munder A, Tümmler B, Pabst R and Tschernig T: Beneficial effects of TLR-2/6 ligation in pulmonary bacterial infection and immunization with Pseudomonas aeruginosa. Inflammation 33: 58-64, 2010 .
25. Knaapen AM, Seiler F, Schilderman PA, Nehls P, Bruch J, Schins RP and Borm PJ: Neutrophils cause oxidative DNA damage in alveolar epithelial cells. Free Radic Biol Med 27: 234-240, 1999.

26. Akpek EK and Gottsch JD: Immune defense at the ocular surface. Eye (Lond) 17: 949-956, 2003.

27. Chang JH, McCluskey PJ and Wakefield D: Toll-like receptors in ocular immunity and the immunopathogenesis of inflammatory eye disease. Br J Ophthalmol 90: 103-108, 2006.

28. Lee HN, Na HK and Surh YJ: Resolution of inflammation as a novel chemopreventive strategy. Semin Immunopathol 35: 151-161, 2013.

29. Taube MA, del Mar Cendra M, Elsahn A, Christodoulides M and Hossain P: Pattern recognition receptors in microbial keratitis. Eye (Lond) 29: 1399-1415, 2015.

30. Hazlett LD: Role of innate and adaptive immunity in the pathogenesis of keratitis. Ocul Immunol Inflamm 13: 133-138, 2005.

31. Hindman HB, Patel SB and Jun AS: Rationale for adjunctive topical corticosteroids in bacterial keratitis. Arch Ophthalmol 127: 97-102, 2009.

32. Herretes S, Wang X and Reyes JM: Topical corticosteroids as adjunctive therapy for bacterial keratitis. Cochrane Database Syst Rev: CD005430, 2014

33. Wilhelmus KR: Indecision about corticosteroids for bacterial keratitis: An evidence-based update. Ophthalmology 109: 835-843, 2002

34. Yoshino T, Nakase H, Honzawa Y, Matsumura K, Yamamoto S, Takeda Y, Ueno S, Uza N, Masuda S, Inui K and Chiba T: Immunosuppressive effects of tacrolimus on macrophages ameliorate experimental colitis. Inflamm Bowel Dis 16: 2022-2033, 2010.

35. Morris MC, Gilliam EA, Button J and Li L: Dynamic modulation of innate immune response by varying dosages of lipopolysaccharide (LPS) in human monocytic cells. J Biol Chem 289: 21584-21590, 2014.

36. Shihab F, Christians U, Smith L, Wellen JR and Kaplan B: Focus on mTOR inhibitors and tacrolimus in renal transplantation: Pharmacokinetics, exposure-response relationships, and clinical outcomes. Transpl Immunol 31: 22-32, 2014.

37. Aomatsu T, Imaeda H, Takahashi K, Fujimoto T, Kasumi E, Yoden A, Tamai H, Fujiyama Y and Andoh A: Tacrolimus (FK506) suppresses TNF- $\alpha$-induced CCL2 (MCP-1) and CXCL10 (IP-10) expression via the inhibition of p38 MAP kinase activation in human colonic myofibroblasts. Int J Mol Med 30: 1152-1158, 2012.

38. Shin MR, Kang SK, Kim YS, Lee SY, Hong SC and Kim EC: TNF- $\alpha$ and LPS activate angiogenesis via VEGF and SIRT1 signalling in human dental pulp cells. Int Endod J 48: 705-716, 2015.

39. Turgut B, Guler M, Akpolat N, Demir T and Celiker U: The impact of tacrolimus on vascular endothelial growth factor in experimental corneal neovascularization. Curr Eye Res 36: 34-40, 2011.

40. Park JH, Joo CK and Chung SK: Comparative study of tacrolimus and bevacizumab on corneal neovascularization in rabbits. Cornea 34: 449-455, 2015.

41. Cavagis A, Takamori E, Granjeiro J, Oliveira R, Ferreira C, Peppelenbosch $M$ and Zambuzzi W: TNF $\alpha$ contributes for attenuating both Y397FAK and Y416Src phosphorylations in osteoblasts. Oral Dis 20: 780-786, 2014

42. Karginov AV, Tsygankov D, Berginski M, Chu PH, Trudeau ED, Yi JJ, Gomez S, Elston TC and Hahn KM: Dissecting motility signaling through activation of specific Src-effector complexes. Nat Chem Biol 10: 286-290, 2014.

43. Fischer L, Saliba F, Kaiser GM, De Carlis L, Metselaar HJ, De Simone P, Duvoux C, Nevens F, Fung JJ, Dong G, et al: Three-year outcomes in de novo liver transplant patients receiving everolimus with reduced Tacrolimus: Follow-up results from a randomized, multicenter study. Transplantation 99: 1455-1462, 2015. 